\title{
In silico investigation of Aedes aegypti male-determining factor (NIX): RNA recognition motif-3, structural model and selective nucleic acid binding mode.
}

Monika A. Coronado a, b*\#, Danilo S. Olivier ${ }^{\mathrm{c}^{*}}$, Kelly C. Borsatto ${ }^{\mathrm{a}}$, Marcos S. Amaral ${ }^{\mathrm{d}}$, Raghuvir K. Arni ${ }^{\mathrm{a}}$, Raphael J. Eberlea,

aultiuser Center for Biomolecular Innovation, Departement of Physics, Instituto de Biociências Letras e Ciências Exatas (Ibilce), Universidade Estadual Paulista (UNESP), São Jose do Rio Preto-SP, 15054-000, Brazil.

b'Institute of Biological Information Processing (IBI-7: Structural Biochemistry), Forschungszentrum Jülich, Jülich, Germany

${ }^{c}$ Federal University of Tocantins, Araguaína-TO, Brazil.

Institute of Physics, Federal University of Mato Grosso do Sul, Campo Grande-MS, Brazil.

* Both authors contributed equally

\# Corresponding authors:

Monika A. Coronado

E-Mail: m.coronado@fz-juelich.de

Raphael J. Eberle

E-Mail: r.eberle@fz-juelich.de 


\begin{abstract}
Mosquito borne viruses and their corresponding human infections have a fast growing impact on worldwide public health systems; in particular, Aedes aegypti and Aedes albopictus are transmitting these viruses. Only female mosquitoes bite and spread diseases. Male development in A. aegypti is initiated by male determining factors. These factors are potential candidates for the implementation of vector control strategies. Where at an early stage in gender development female mosquitoes are converted into harmless males. Among these factors, a novel gene, NIX, has been identified that shares moderate identity with transformer-2, one of the key sex-determination genes in Drosophila melanogaster. Hall et al. 2015 described two RNA recognition motifs (RRMs) in the NIX sequence, but a UniProt database search showed that $A$. aegypti NIX contains three RNA RRM. A homology model of NIX_RRM-3 was generated to investigate the interaction with RNA using molecular dynamics simulations. The sequence AGACGU was the most interesting result. During the binding process A1, G2 and G5 adopt an unusual syn conformation. The results let assume that the C-terminus of NIX_RRM-3 is involved in the recognition process of the target RNA motif (e.g. AG) and controls the interaction between target RNA and A. aegypti NIX.
\end{abstract}

Keywords: Aedes aegypti, male-determining factor, RNA recognition motif, RNA, molecular dynamics. 


\section{Introduction}

Climate change has a profound effect on the global occurrence and burden of infectious diseases [1-3], especially mosquito borne viruses. The resulting altered distribution of viruses such as Dengue, Yellow Fever, West Nile, Chikungunya, Zika and Japanese Encephalitis and their vectors (e.g. Aedes aegypti and Aedes albopictus) are key factors of public health that can dramatically change infection patterns and require attention [4-6].

The recent strategies to control mosquitoes that transmit viruses are based on genetic techniques such as the sustained release of sterile or transgenic "self-limiting" mosquitoes [7]. Given the fact that only female mosquitoes bite and spread diseases, there has been substantial interest in manipulating mosquito sex determination using these genetic techniques and others, including gene drive $[8,9]$. Therefore, elucidating the genetic basis for sex determination could, for instance, facilitate the male-only production of cohorts for release, or allow transformation of mosquitoes with sex-specific "self-limiting" gene cassettes [10].

Male development in Aedes aegypti (A. aegypti) is initiated by male factors (M-factor) located on the homomorphic sex-determining chromosome within a Y chromosome-like region referred to as the M-locus [11,12]. Consequently, a mosquito M-factor would be useful in implementing vector control strategies where female mosquitoes at an early stage in gender development are converted into harmless males [13]. A number of malespecific genomic sequences were identified in A. aegypti [14]. Among these, a novel gene classified as Nix that shares moderate identity with transformer-2, one of the key sexdetermination genes in D. melanogaster [15]. The A. aegypti Nix gene is $985 \mathrm{bp}$ in length and encodes a polypeptide with 288 amino acids including two RNA recognition motifs (RRMs). Nix exhibits persistent male linkage and is expressed early in embryonic development [14], being essential characteristics of the M-factor. Furthermore, somatic knockout of Nix in A. aegypti male embryos results in feminization whereas ectopic expression of Nix in female embryos leads to masculinization, clearly demonstrating that Nix is instrumental for the initiation of male development [14]. Nix is hypothesized to be a splicing factor that acts directly or indirectly on the doublesex (dsx) and fruitless (fru) genes involved in sex-determination [16].

So far, no structural information is available about the A. aegypti Nix protein and its interaction with the target RNA. The investigation of the binding specificity between protein and nucleic acids can help to understand the interaction process and 
conformational changes in the protein and the RNA, respectively.

Analyses of the UniProt database (https://www.uniprot.org/) demonstrates that the $A$. aegypti NIX protein sequence contains an additional RRM to the two RRMs described previously [14]. A model of the full-length protein and the three RRM domains were constructed. Molecular dynamics (MD) simulations were performed with the NIX_RRM3 domain to observe the stability of the model over time. The NIX_RRM-3 model was used for docking and MD simulations with a set of various RNA sequences with the goal of identifying an appropriate target RNA sequence and exploring the binding process between the protein and the RNA. AGACGU showed a robust interaction with NIX RRM-3. Interestingly A1, G2 and G5 bases undergo an unusual syn conformation during the interaction with the C-terminus of NIX_RRM-3. We suppose that the NIX_RRM-3 domain in NIX is essential for identification and binding of the target RNA.

\section{Material and Methods}

\subsection{In silico analysis}

Multiple sequences of NIX, male factor, transformer, and RNA binding proteins were retrieved from the NCBI database and sequence alignments were performed using the MUSCLE [17] and Box Shade (http://www.ch.embnet.org/software/BOX_form.htm) web servers. A homology model of full-length A. aegypti NIX (GeneBank: AHW46195.1 Uniprot: A0A0F6MY85) as well as A. aegypti NIX_RRM-1,-2, and -3 domains were created using the Zhang lab I-tasser online tool [18].

\subsection{Docking and molecular dynamics}

The HADDOCK2.2 [19] web server was used to perform the molecular docking between the A. aegypti NIX_RRM-3 protein domain and the following RNA sequences (AAGAAC, AGACGU and AAACGU). To choose the start configuration, energy values for the protein-RNA interaction as well as visual inspection was used. Molecular dynamics simulations were performed using the Amber18 [20] software package. The descriptions of all-atoms interactions were performed using the FF14SB [21] force field for the protein while the RNA sequences were described using the OL3 [22] force field. The NIX_RRM-3 model was created using the Zhang lab I-tasser online tool [1819]. The protonation state for the NIX_RRM-3 protein was settled using $\mathrm{H}++$ [23] web server at $\mathrm{pH}$ 7.5. The initial protein model was submitted to MD simulations for $300 \mathrm{~ns}$ to obtain 
a relaxed structure and the model quality monitored by PROCHECK [24]. NIX_RRM-3 and the protein/RNA-complexes were neutralized with $\mathrm{Na}^{+}$or $\mathrm{Cl}^{-}$ions and, placed in an octahedral box with TIP3P water extended $10 \AA$ from the protein atoms. Energy minimization was performed in two steps to remove bad contacts. Initially, both the protein and the protein/RNA complex were constrained and a constant force of 50.0 $\mathrm{kcal} / \mathrm{mol}-\AA^{2}$ was performed for 5000 steps of steepest descent succeeded by 5000 steps of conjugate gradient, following which, 10000 steps energy minimization without constraints were conducted. The system was heated from 0 to $298 \mathrm{~K}$ under constant atom number, volume and temperature (NVT) ensemble, maintaining the protein and protein/RNA complex restrained with a constant force of $10.0 \mathrm{kcal} / \mathrm{mol}-\AA^{2}$. Thereafter, an equilibration step was performed under constant atom number, pressure and temperature (NPT) ensemble for 500 ps and the simulation was performed for $200 \mathrm{~ns}$ with 2 fs time steps. Constant temperature $(298 \mathrm{~K})$ and pressure (1 atm) were controlled by Langevin coupling. Long-range electrostatic interactions were treated via Particle-Mesh Ewald (PME) [25] while a cut-off distance of $10 \AA$ was attributed to Van der Waals interactions.

\subsection{Molecular dynamics analyses and Energy Interaction calculations}

The MD simulations were analyzed using CPPTRAJ [26] of the AmberTools18 package. System equilibration and convergence were assessed by analysis of the root mean square deviations (RMSDs). Protein and RNA flexibilities were studied by root mean square fluctuation (RMSF). RMSFs were calculated residue-by-residue over the equilibrated trajectories. Radius of Gyration (RoG) and surface area were calculated to check major structural changes in the protein. The molecular mechanics/generalized Born surface area (MM/GBSA) energy was calculated between the protein and the RNA molecules considering the stable regime using the last $20 \mathrm{~ns}$ of the MD simulations and stripping all the ions and water molecules.

\section{Results and Discussion}

\subsection{Sequence analysis of full length $A$. aegypti NIX protein and RRM domains 1-3}

A. aegypti NIX is a 288 amino acid polypeptide that has been reported of comprising two RNA recognition motifs (RRMs). The first RRM is comprised of the region Tyr19 to Ser94 and the second RRM of the region Arg205 to Lys282 [14]. However, a search on the UniProt database (https://www.uniprot.org/), demonstrated that A. aegypti NIX, 
contains a third RRM motif (Ile108 to Arg179). The schematic construction of the NIX protein with RRM-1 to RRM-3 are presented in Fig. 1A and 1B.

Figure 1

Fig. 1. Primary and tertiary structures of A. aegypti NIX. (A) Schematic diagram of A. aegypti Nix. The three RRM domains are represented in different colors (RRM-1 (blue), RRM-2 (green), and RRM-3 (orange)) and, amino acid residue numbers indicate the approximate boundaries of the three RRMs. (B) Homology model of Nix, the RRM domains; color coded as in A. (C) Sequence alignment of NIX_RRM-1 to RRM-3 with different RRMs. RNP1 and RNP2 motifs are highlighted (purple and red, respectively). The sequence of NIX_RRM-1 is aligned with Xenopus laevis Embryonic polyadenylate-binding protein 2-B (ePABP2), RNA-binding motif-containing protein 1 (ZCRB1), Homo sapiens Polyadenylate-binding protein 1 (PABPC1), Saccharomyces cerevisiae U4/U6 snRNA-associatedsplicing factor (PrP24). The NIX_RRM-2 sequence is aligned with Homo sapiens Polyadenylate-binding protein 1 (PABPC1), Saccharomyces cerevisiae RNA-binding protein (MIP6), Mesocricetus auratus Nucleolin (NCL), Saccharomyces cerevisiae U4/U6 snRNA-associated-splicing factor (PrP24). The NIX_RRM-3 sequence (orange box) is aligned with Saccharomyces cerevisiae U4/U6 snRNA-associated-splicing factor (PrP24), Homo sapiens U1 small nuclear ribonucleoprotein A (SNRPA), Homo sapiens Polyadenylate-binding protein 1 (PABPC1), Homo sapiens Transformer-2 protein homolog beta (TRA2B). The amino acids involved in nucleic acid binding and the corresponding bases from PABPC1 and TRA2B crystal structures (PDB code: 1CVJ, 2RRA and 2KXN) are highlighted, adenine (A), guanine $(\mathrm{G})$ and phosphate backbone $(\mathrm{P})$.

A typical RRM domain consists of 80 to 90 amino acid residues [27]. However, the A. aegypti NIX_RRMs are slightly smaller whereas RRM-1 comprises 75 residues, RRM2 of 71 and RRM-3 of 77 respectively. Nevertheless, all three RRMs domains contain the characteristic RNA nucleoprotein motifs 1 and 2 (RNP1 and RNP2) as indicated in Fig. 1C. The RNP motifs in the RRMs are necessary for their specific RNA-recognition modes [27]. The consensus RNP2 and RNP1 sequences are defined as [I/L/V]-[F/Y]-[I/LV]-X$\mathrm{N}-\mathrm{L}$ and $[\mathrm{K} / \mathrm{R}]-\mathrm{G}-[\mathrm{F} / \mathrm{Y}]-[\mathrm{G} / \mathrm{A}]-[\mathrm{F} / \mathrm{Y}]-[\mathrm{I} / \mathrm{L} / \mathrm{V}]-\mathrm{X}-[\mathrm{F} / \mathrm{Y}]$ [27]. Based on the sequence alignment of the three NIX_RRMs and related protein structures we identified the $A$. aegypti NIX_RRM-1 (RNP2 and RNP1) motifs sequences are I-Y-I-G-N-I and V-K-PA-I-V-R-Y, respectively. NIX_RRM-2 presents the motif sequences of V-Y-I-N-D-K and N-N-M-I-Y-I-N-F for RNP2 and RNP1, respectively. The sequences for NIX_RRM3 (RNP2 and RNP1) were identified as I-G-I-F-G-L and K-Q-Y-G-F-I-Y-Y, respectively (Fig. 1C). The A. aegypti NIX RNP2s and RNP1s differs from the typical consensus sequences described by Muto and Yokoyama (2012) [27]. NIX RNP2 and RNP1 sequences of RRM-3 domain showed a high similarity with the corresponding RNPs of Homo sapiens Transformer-2 protein homolog beta (TRA2B), with 3D structures in complex with RNA (PDB codes: 2RRA and 2KXN).

\subsection{A. aegypti NIX_RRM-3 model generation, molecular dynamics simulation and validation of the predicted structure}

The NIX_RRM-3 model was generated using the Zhang lab I-tasser online tool [18]. 
Molecular dynamics (MD) simulations were performed to investigate the structural and dynamical changes over time in the NIX_RRM-3 model. The equilibration of the simulations was monitored by calculating the root mean square deviation (RMSD) and the root mean square fluctuation (RMSF) for the backbone atoms with respect to the initial conformation over time with the aim of verifying that all simulations maintained confirmations that were stable during an 300 ns simulation (Supplementary Fig. S1). The NIX_RRM-3 model after MD simulations is presented in Fig. 2A.

The evaluation of the stereochemical properties of the predicted A. aegypti NIX_RRM-3 model was performed using PROCHECK [24]. The corresponding Ramachandran plot demonstrates that the conformations of $90 \%$ of the residues are located in the favored regions, $9.0 \%$ are in the allowed regions and only $1.0 \%$ were flagged as outliers (Fig. 2B). The stereochemical parameters for the omega angle is -1.3 , bad contact are -0.4 and the overall G-factor is 0.3 . The final model consists of 83 residues. The structural overlay of the NIX_RRM-3 model and the related structure of the human TRA2B (PDB entry: 2KXN) (RMSD $2.85 \AA$ ) is presented in Fig. 2C and supplementary Fig. 2.

Figure 2

Fig. 2. 3D model of A. aegypti NIX_RRM-3. (A) Homology model after MD simulations of NIX_RRM-3 domain. (B) Ramachandran plot of NIX_RRM-3 after MD simulations. (C) Structural overlay of NIX_RRM-3 (orange) with human TRA2B (PDB entry: 2KXN) (yellow). (D) Scheme of the NIX_RRM-3 $\beta$-sheet distribution, some of the amino acids which form the RNP2 ( $\beta 1)$ and RNP2 ( $\beta 3$ ) are highlighted (asterisk).

The canonical RRM structure consists of a four-stranded antiparallel $\beta$-sheet packed against two $\alpha$-helices with a typical $\beta \alpha \beta \beta \alpha \beta$ topology [27] whereas the four-stranded $\beta$ sheet is the primary RNA-binding surface [28]. The modeled domains of NIX_RRM-1 and -3 contain an additional $\beta$-strand, which is located in the loop between $\alpha$-helix 2 and the C-terminal $\beta$-strand. NIX_RRM-2 possesses the typical topology of the RRM family (Supplementary Fig. S3 and Fig. 1B). The human TRA2B and Polyadenylate-binding protein 1 (PABPC1) protein structures contain also an additional $\beta$-strand at the same position as the NIX_RRM-3 model [29,30] (Fig. 2C and supplementary Fig. S2). Human TRA2B and PABPC1 structures in complex with RNA showed that the nucleic acids mainly interact with the core $\beta \alpha \beta \beta \alpha \beta$ and the additional $\beta$-strand seems to have a stabilizing function for the RNA interaction [29,30] (Supplementary Fig. S4). The core of the $\beta \alpha \beta \beta \alpha \beta$ topology is formed by $\beta 1$ and $\beta 3$; RNP2 and RNP1 are located in these $\beta$ strands, which are also shown for NIX_RRM-3 (Fig. 2C and D). 


\subsection{Identification of a target RNA sequence interacting with the $A$. aegypti NIX_RRM-3 model}

A computational approach was used a) to identify a target RNA sequence that interacts with NIX_RRM-3, and b) to study their corresponding interactions. For this, a RNA sequence was inserted near the RNP1 and RNP2 motifs of NIX_RRM-3 using molecular docking. Subsequent MD simulations were carried out to provide statistical results to better understand the interactions between the RRM-3 domain and the RNA. It has been suggested that the RNA sequence AAGAAC is utilized to interact with human TRA2B (PDB entry: 2KXN). Based on the similarities between NIX_RRM-3 and TRA2B RNPs, the RNA sequence was used for a primary MD simulation with NIX_RRM-3. The analysis of the RMSD and RMSF showed a strong fluctuation during 200 ns of MD simulations (Supplementary Fig. S5) and the RNA molecule shifted $>20 \AA$ away from the starting position (Table 1 and supplementary Fig. S9). Based on those primary results the RNA sequence AGACGU was chosen for further MD simulations with NIX_RRM-3. The human TRA2B recognize mainly AGAA of AAGAAC [29] and Auweter et al. (2006) suggested a default RNA binding sequence for an RRM that might be a dinucleotide A/C-G in N1-N2 position [28]. The first three bases of AGACGU are similar to the AGAA sequence recognized by human TRA2B and the last three bases contain the CG pattern postulated by Auweter et al. (2006). Interestingly the RMSF fluctuation of the NIX_RRM-3/AGACGU complex reduced dramatically (Supplementary Fig. S6) compared with the NIX_RRM-3/AAGAAC complex (Supplementary Fig. S5).

Table 1. RNA sequences used for MD simulations with A. aegypti NIX_RRM-3 model.

\begin{tabular}{lll}
\hline RNA sequence & Distance start-final position [̊] & General information MD simulation \\
\hline AAGAAC & $>20$ & Supplementary Fig. S5 and S9 \\
AGACGU & $\sim 4$ & Supplementary Fig. S6 and S9 \\
AGACGU* & $>20$ & Supplementary Fig. S7 and S9 \\
AAACGU & $>20$ & Supplementary Fig. S8 and S9 \\
\hline
\end{tabular}

* MD simulations of truncated NIX_RRM-3 model.

Analysis of the NIX_RRM-3/AGACGU complex demonstrated that the RNA bases A1 and G2 (GACGU) interact mainly with Arg73 and Glu77 (Fig. 3A and C), residues that are located in the unstructured C-terminus of the protein. Evaluation of the RMSF of NIX_RRM-3 and NIX_RRM-3/AGACGU complex showed that the movement of the Cterminus is reduced significantly upon RNA binding. To investigate the interactions between the RNA and the C-terminus of the NIX_RRM-3 model the protein was truncated, the last eight C-terminal residues (PEKKVFKN) were removed, and 
subsequent MD simulations with AGACGU were performed. Interestingly, the RMSF of the truncated complex increased compared with the full-length NIX_RRM-3/AGACGU complex (Supplementary Fig. S7) with the RNA sequence (AGACGU) moving > $20 \AA$ away from the starting position. These results demonstrate the importance of the protein C-terminus for the interaction with RNA. Additionally, the importance of G2 (A $\underline{G A C G U}$ ) for the interaction with NIX_RRM-3 was investigated, thus guanine (G2) was replaced by adenine and new MD simulations were performed, the analysis indicated the formation of an unstable complex (RRM-3/AAACGU) (Table 1 and supplementary Fig. S8).

\subsection{AGACGU interaction with $A$. aegypti NIX_RRM-3 model}

The interaction of AGACGU with NIX_RRM-3 induces secondary structural changes in the protein model. The $\alpha$-helix content increased from 15 to $22 \%$ and the $\beta$-strand content increased from 28 to $32 \%$ respectively whereas the random coiled amount decreased from 40 to $29 \%$ in the model of the NIX_RRM-3 complex compared to the unbound protein. Upon RNA binding, an additional $\beta$-strand was formed and the RNP1 containing $\beta-3$ in the free protein becomes $\beta-4$ in the protein/RNA complex (Supplementary Fig. S10A and B).

Figure 3

Fig. 3. A. aegypti NIX_RRM-3/AGACGU complex after MD simulations. (A) NIX_RRM-3/AGACGU complex, protein in ribbon view and the RNA is presented as sticks. (B) NIX_RRM-3/AGACGU complex in a Coloumbic surface representation, positive surface in blue and negative surface in red, RNA is in sticks. (C) Interaction of AGACGU with NIX_RRM-3, single base interactions are shown. The amino acids involved in hydrogen bonds and intramolecular hydrogen bonds between the RNA bases are highlighted (red dotted lines) and hydrophobic interactions are emphasized by orange bands.

AGACGU also undergoes conformational changes during the interaction with NIX_RRM-3 (Supplementary Fig. S10C and D). Amino acid residues interacting with AGACGU are Phe5, Lys40, Gln41, Arg73 and Glu77 as indicated in Fig. 3A-C.

Interestingly, three bases in the AGACGU sequence change conformation upon binding to NIX_RRM-3. A1, G2 and G5 adopted an unusual syn conformation (Fig. 4); A1 is connected by hydrogen bonds with Arg73 and Glu77 and an intramolecular hydrogen bond with A3. G2 interact with Glu77 and with the sugar moiety of A1. G5 is stabilized via hydrophobic interactions with Phe 5 of the protein and with intramolecular hydrogen bonds of the A3 and C4 sugar moieties (Fig. 3C). 


\section{Figure 4}

Fig. 4. Conformational changes of the AGACGU nucleotide bases after binding to NIX_RRM-3. A1, G2 and G5 changed from anti to syn conformation. The red sphere demonstrate the rotation of the $\chi$ angle.

The syn conformation of RNA bases in RNA/RRM complexes occur in several examples, A4 in the 5'-AGAA-3' sequence interacting with human TRA2B [29]; A2 of the 5'-CAUC-3' RNA sequence bound by the Srp RRM [31]; A12 from the 5'-CAA3 'triplet interacting with RBMY RRM [32]; guanines are noted to adopt a syn conformation during binding with RRM proteins [33-35]. A default RNA binding sequence for an RRM that might be a dinucleotide $\mathrm{A} / \mathrm{C}-\mathrm{G}$ in $\mathrm{N} 1-\mathrm{N} 2$ position has been reported [28]; this is the same sequence, which we observed for the AGACGU interaction with NIX_RRM-3.

Human TRA2B and PABPC1 protein structures in complex with RNA suggested that RNA mainly interacts with the core $\beta \alpha \beta \beta \alpha \beta$, formed by $\beta$-strand 1 and 3 , both carrying RNP2 and RNP1 [29,30]. In this region, a typical sequence of aromatic residues in RRM proteins can be observed which are involved in the interaction with the RNA. The aromatic amino acid residues on the $\beta 1$ and $\beta 4$ surface Phe5, Tyr42, Phe44 and Tyr46 of NIX_RRM-3 show similarities compared with that of human TRA2B (Fig.5A and B).

\section{Figure 5}

Fig. 5. Aromatic residues in the $\beta \alpha \beta \beta \alpha \beta$ core of RRM proteins that are involved in the RNA interaction. Top panel: Schematic representation of the aromatic amino acids in $\beta$-sheet 1 and $\beta$-sheet 3/4. Asterisk: After RNA interaction with NIX_RRM-3 an additional $\beta$-sheet is formed and $\beta-3$ numeration change to $\beta-4$. Down panel: tertiary structures of RRMs with RNA. (A) NIX_RRM-3. (B) Human TRA2B (PDB entry: 2KXN).

Comparison of the aromatic amino acid sequence elements of the human TRA2B in $\beta 1$ and $\beta 3$ with the NIX_RRM-3 model showed that Phe161 is replaced by Tyr42 (Fig. 5A and B). Tyr 42 in NIX_RRM-3 seems to have an important function in the stabilization of the RNA binding region while the residues of the C-terminus (Arg73 and Glu77) seem to interact with A1 and G2. Both residues fluctuate in the NIX_RRM-3 model (Fig. 6A).

\section{Figure 6}

Fig. 6. Interaction network between aromatic residues in the $\beta \alpha \beta \beta \alpha \beta$ core of NIX_RRM-3 and amino acids involved in the interaction with AGACGU. (A) Tertiary structure of NIX_RRM-3. (B) Tertiary structure of NIX_RRM3/AGACGU complex without the RNA sequence. (C) Interaction surface of NIX_RRM-3/AGACGU complex; the aromatic residues of the $\beta \alpha \beta \beta \alpha \beta$ core and the amino acids involved in the interaction with AGACGU are colored (orange).

Interestingly, after interacting with AGACGU, the C-terminal residue Arg73 interacts 
mainly with A1 and Glu77 with both bases (A1 and G2). Furthermore, Arg73 and Glu77 form bifurcated hydrogen bonds with each other and one with Tyr42 with a distance range of 2.7 to $3.2 \AA$ to stabilize the protein/RNA complex (Fig. 6B).

Fig. 6C demonstrates the surface of interaction of the NIX_RRM-3/AGACGU complex. It is likely that the interaction network between NIX_RRM-3 residues Tyr42, Arg73 and Glu77 forms a specific binding region for AG RNA sequences. We suggest that the C-terminus of NIX_RRM-3 is important to identify specific RNA sequences. When RRMs bind to the target RNA, N- and C-terminus of the protein may be involved in recognition and binding processes, which is typical for RRM proteins [36-38].

\section{Conclusion}

The spread of virus diseases (Dengue, Zika, Yellow fever and Chikungunya virus) transmitted by mosquitoes such as Aedes aegypti is an event that is occurring with greater frequency in warmer, developing countries. Low investment in fragile health systems generates a perfect setting for infectious diseases. Global climatic, environmental and ecological changes provide for the mosquitoes a favorable environment by breaking their natural and ecological boundaries and becoming established in new geographic locations. The lack of an effective antiviral treatments or vaccines for Dengue, Zika, Yellow fever and Chikungunya virus infections means that vector control is a highly attractive, alternate strategy to safeguard populations against infections. However, the efficacy of insecticide-based vector control is limited due to elevated levels of insecticide resistance in vector insect populations, lending further urgency to efforts in developing novel vector control strategies $[39,40]$. One promising approach, sterile insect technique (SIT), has been successfully used in past insect pest eradication programs. The new world screwworm (Cochliomyia hominivorax), the mediterranean fruit fly (Ceratitis capitate), and the tsetse fly (Glossina morsitans) have been successfully targeted with this technique [41-43].

There has been substantial interest in the manipulation of mosquito sex development since only female mosquitoes spread viral diseases during a blood meal. Male development in $A$. aegypti is initiated by male factors and these factors would be useful in implementing vector control strategies where developing female mosquitoes are converted into males. A new identified A. aegypti gene, NIX, possess typical characteristics of a male factor [14]. To date, no detailed information is available about the A. aegypti NIX interaction with the target RNA on the molecular level. A. aegypti 
NIX contain three RNA recognition domains and NIX_RRM-3 contains an extended Cterminal tail when compared with NIX_RRM-1 and -2. We suggest that this tail is important for the recognition of a motif in the target RNA, where the C-terminus scans the RNA until the target motif, AG, is recognized. This interaction induces conformational changes and the three RRM domains of $A$. aegypti NIX bind to the target RNA (Fig. 7).

\section{Figure 7}

Fig. 7. Suggested mode of recognition and interaction of $A$. aegypti NIX with target RNA. NIX_RRM1 (blue), NIX_RRM-2 (green), NIX_RRM-3 (orange), RNPs (black circles) and RNA (grey). (A) A. aegypti NIX and free target RNA. (B) C-terminus of NIX_RRM-3 scan the target RNA sequence for a defined recognition motif (e.g. AG). (C) After recognition of the RNA motif, the interaction of NIX_RRM-3 RNP1 and RNP2 with the target RNA can induce conformational changes in the protein and NIX_RRM-2; RRM-1 could bind to the RNA. 


\section{Conflict of interest}

Authors declare that there exists no conflict of interest.

\section{Aknowledgements}

We wish to thank Prof. Dr. D. Willbold, Dr. M. Schwarten, Dr. S. Feuerstein and N. Bleffert for useful discussion and for revising the manuscript. This research was supported by grants from CNPq [Grant numbers 309940/2019-2, 435913/2016-6, 401270/2014-9, 307338/2014-2], FAPESP [Grant numbers 2016/129040, 2018/07572-3, 2018/126590, 2019/05614-3], Fundect [23/200.307/2014], CAPES and PROPe UNESP.

\section{Author contributions}

M.A.C., R.J.E and K.C.B performed the In silico analysis of NIX and NIX_RRM-3. D.S.O. and M.S.A. performed the molecular docking and MD simulations. M.A.C. and R.J.E analyzed the computational experiments. R.K.A. guided the research. M.A.C. and R.J.E coordinated the research and planned all the experiments. M.A.C. and R.J.E wrote the manuscript and all authors read and took part in revising the final version.

\section{References}

[1] Hoberg, E.P.; Brooks, D.R. Evolution in action: climate change, biodiversity dynamics and emerging infectious disease. Phil. Trans. R. Soc. B. 2015, 370, 20130553.

[2] Lafferty, K.D. The ecology of climate change and infectious diseases. Ecology. 2009, 90, 888-900.

[3] Escobar, L.E.; Romero-Alvarez, D.; Leon, R.; Lepe-Lopez, M.A.; Craft, M.E.; Borbor-Cordova, M.J.; Svenning, J.C. Declining Prevalence of Disease Vectors Under Climate Change. Sci Rep. 2016, 6, 39150.

[4] Messina, J.P.; Brady, O.J.; Pigott, D.M.; Golding, N.; Kraemer, U.; Scott, T.W.; Wint, G.R.; Smith, D.L.; Hay, S.I. The many projected futures of dengue. Nat. Rev. Microbiol. 2015, 13, 230-239. 
[5] Campbell, L.P.; Luther, C.; Moo-Llanes, D.; Ramsey, J.M.; Danis-Lozano, R.; Peterson, A.T. Climate change influences on global distributions of dengue and chikungunya virus vectors. Phil. Trans. R. Soc. B. 2015, 370, 20140135.

[6] Carlson, C.J.; Dougherty, E.R.; Getz, W. An ecological assessment of the pandemic threat of Zika virus. PLoS Negl. Trop. Dis. 2016, 10, e0004968.

[7] Alphey, L. Genetic control of mosquitoes. Annu. Rev. Entomol. 2014, 59, 205-24.

[8] Gilles, J.R.L.; Schetelig, M.F.; Scolari, F.; Marec, F.; Capurro, M.L.; Franz, G.; Bourtzis, K. Towards mosquito sterile insect technique programmes: exploring genetic, molecular, mechanical and behavioural methods of sex separation in mosquitoes. Acta Trop. 2014, 132, S178-87.

[9] Hoang, K.P.; Teo, T.M.; Ho, T.X.; Le, V.S. Mechanisms of sex determination and transmission ratio distortion in Aedes aegypti. Parasit Vectors. 2016, 9, 49

[10] Turner, J.; Krishna, R.; Van’t Hof, A.E.; Sutton, E.R.; Matzen, K.; Darby, A.C. The sequence of a male-specific genome region containing the sex determination switch in Aedes aegypti. Parasit Vectors. 2018, 11, 549.

[11] Gilchrist, B.M.; Haldane, J.B.S. Sex linkage and sex determination in a mosquito, Culex molestus. Hereditas. 1947, 33, 175-190.

[12] Newton, M.E.; Wood, R. J.; Southern, D. I. Cytological mapping of the M and D loci in the mosquito, Aedes aegypti (L.). Genetica. 1978, 48, 137-143.

[13] Papathanos, P.A.; Bossin, H.C.; Benedict, M.Q.; Catteruccia, F.; Malcolm, C.A.; Alphey, L.; Crisanti, A. Sex separation strategies: past experience and new approaches. Malar. J. 2009, 8, S5.

[14] Hall, A.B.; Basu, S.; Jiang, X.; Qi, Y.; Timoshevskiy, V.A.; Biedler, J.K.; Sharakhova, M.V.; Elahi, R.; Anderson, M.A.E.; Chen, X.G.; Sharakhov, I.V.; Adelman, Z.N.; Tu, Z. A male-determining factor in the mosquito Aedes aegypti. Science. 2015, $348,1268-1270$.

[15] Salz, H.K.; Erickson, J.W. Sex determination in Drosophila: The view from the top. Fly (Austin). 2010, 4, 60-70.

[16] Adelman, Z.N.; Tu, Z. Control of mosquito-borne infectious diseases: sex and gene 
drive. Trends Parasitol. 2016, 32, 219-229.

[17] Edgar, R.C. MUSCLE: multiple sequence alignment with high accuracy and high throughput. Nucleic Acids Res. 2004, 32, 1792-1797.

[18] Zhang, Y. I-TASSER server for protein 3D structure prediction. BMC bioinformatics. 2008, 9, 40.

[19] Van Zundert, G.C.P.; Rodrigues, J.P.G.L.M.; Trellet, M.; Schmitz, C.; Kastritis, P.L.; Karaca, E.; Melquiond, A.S.J.; van Dijk, M.; de Vries, S.J.; Bonvin, A.M.J.J. The HADDOCK2.2 Web Server: User-Friendly Integrative Modeling of Biomolecular Complexes. J. Mol. Biol. 2016, 428, 720-725.

[20] Case, D.A.; Cerutti, D.S.; Cheatham III, T.E.; Darden, T.A.; Duke, R.E.; Giese, T.J.; Gohlke, H.; Goetz, A.W.; Greene, D.; Homeyer, N.; Izadi, S.; Kovalenko, A.; Lee, T.S.; LeGrand, S.; Li, P.; Lin, C.; Liu, J.; Luchko, T.; Luo, R.; Mermelstein, D.; Merz, K.M.; Monard, G., Dmy, H.; Pak, H. AMBER. University of California, San Francisco. 2017.

[21] Maier, J.A.; Martinez, C.; Kasavajhala, K.; Wickstrom, L.; Hauser, K.E.; Simmerling, C. ff14SB: Improving the Accuracy of Protein Side Chain and Backbone Parameters from ff99SB. J. Chem. Theory Comput. 2015, 11, 3696-3713.

[22] Zgarbová, M.; Otyepka, M.; Šponer, J.; Mládek, A.; Banáš, P.; Cheatham III, T.E.; Jurečka, P. Refinement of the Cornell et al. Nucleic acids force field based on reference quantum chemical calculations of glycosidic torsion profiles. J. Chem. Theory Comput. 2011, 7, 2886-2902.

[23] Gordon, J.C.; Myers, J.B.; Folta, T.; Shoja, V.; Heath, L.S.; Onufriev, A. H++: a server for estimating pKas and adding missing hydrogens to macromolecules. Nucleic Acids Res. 2005, 33(Web Server), W368-371.

[24] Laskowski, R.A.; MacArthur, M.W.; Moss, D.S.; Thornton, J.M. PROCHECK - a program to check the stereochemical quality of protein structures. J. App. Cryst. 1993, 26, 283-291.

[25] Darden, T., York, D., Pedersen, L. Particle mesh Ewald: An N· $\log (\mathrm{N})$ method for Ewald sums in large systems. J. Chem. Phys. 1993, 98, 10089.

[26] Roe, D.R.; Cheatham III, T.E. PTRAJ and CPPTRAJ: software for processing and analysis of molecular synamics trajectory data. J. Chem. Theory Com. 2013, 9, 3084- 
3095 .

[27] Muto, Y.; Yokoyama, S. Structural insight into RNA recognition motifs: versatile molecular Lego building blocks for biological systems. Wiley Interdiscip. Rev. RNA. 2012, 3, 229-246.

[28] Auweter, S.D.; Oberstrass, F.C.; Allain, F.H.T. Sequence-specific binding of singlestranded RNA: is there a code for recognition? Nucleic Acids Res. 2006, 34, 4943-4959.

[29] Cléry, A.; Jayne, S.; Benderska, N.; Dominguez, C.; Stamm, S.; Allain, F.H. Molecular basis of purine-rich RNA recognition by the human SR-like protein Tra2- $\beta 1$. Nat. Struct. Biol. 2011, 18, 443.

[30] Deo, R.C.; Bonanno, J.B.; Sonenberg, N.; Burley, S.K. Recognition of polyadenylate RNA by the poly (A)-binding protein. Cell. 1999, 98, 835-845.

[31] Hargous, Y.; Hautbergue, G.M.; Tintaru, A.M.; Skrisovska, L.; Golovanov, A.P.; Stevenin, J.; Lian, L.Y.; Wilson, S.A.; Allain, F.H. Molecular basis of RNA recognition and TAP binding by the SR proteins SRp20 and 9G8. EMBO J. 2006, 25, 5126-5137.

[32] Skrisovska, L.; Bourgeois, C.F.; Stefl, R.; Grellscheid, S.N.; Kister, L.; Wenter, P.; Elliott, D.J.; Stevenin, J.; Allain, F.H. The testis-specific human protein RBMY recognizes RNA through a novel mode of interaction. EMBO Rep. 2007, 8, 372-379.

[33] Allain, F.H.T.; Bouvet, P.; Dieckmann, T.; Feigon, J. Molecular basis of sequencespecific recognition of pre-ribosomal RNA by nucleolin. EMBO J. 2000, 19, 6870-6881. [34] Ding, J.; Hayashi, M.K.; Zhang, Y.; Manche, L.; Krainer, A.R.; Xu, R.M. Crystal structure of the two-RRM domain of hnRNP A1 (UP1) complexed with single-stranded telomeric DNA. Genes dev. 1999, 13, 1102-1115.

[35] Enokizono, Y.; Konishi, Y.; Nagata, K.; Ouhashi, K.; Uesugi, S.; Ishikawa, F.; Katahira, M. Structure of hnRNP D complexed with single-stranded telomere DNA and unfolding of the quadruplex by heterogeneous nuclear ribonucleoprotein D. J. Biol. Chem. 2005, 280, 18862-18870.

[36] Tsuda, K.; Kuwasako, K.; Takahashi, M.; Someya, T.; Inoue, M.; Terada, T.; Kobayashi, N.; Shirouzu, M.; Kigawa, T.; Tanaka, A.; Sugano, S.; Güntert, P.; Muto, Y.; Yokoyama, S. Structural basis for the sequence-specific RNA-recognition mechanism of human CUG-BP1 RRM3. Nucleic Acids Res. 2009, 37, 5151-5166. 
[37] Ding, J.; Hayashi, M.K.; Zhang, Y.; Manche, L.; Krainer, A.R.; Xu, R.M. Crystal structure of the two-RRM domain of hnRNP A1 (UP1) complexed with single-stranded telomeric DNA. Genes Dev. 1999, 13, 1102-1115.

[38] Cléry, A.; Blatter, M.; Allain, F.H. RNA recognition motifs: boring? Not quite. Curr. Opin. Struct. Biol. 2008, 18, 290-298.

[39] Wilder-Smith, A.; Gubler, D.J.; Weaver, S.C.; Monath, T.P.; Heymann, D.L.; Scott, T.W. Epidemic arboviral diseases: priorities for research and public health. Lancet Infect. Dis. 2017, 17, e101-e106.

[40] Rather, I.A.; Parray, H.A.; Lone, J.B.; Paek, W.K.; Lim, J.; Bajpai, V.K.; Park, Y.H. Prevention and Control Strategies to Counter Dengue Virus Infection. Front. Cell. Infect. Microbiol. 2017, 7, 336.

[41] Wyss, J.H. Screwworm eradication in the Americas. Ann. NY Acad. Sci. 2000, 916, 186-193.

[42] Hendrichs, J.; Robinson, A.; Cayol, J.; Enkerlin, W. Medfly areawide sterile insect technique programmes for prevention, suppression or eradication: the importance of mating behavior studies. Fla. Entomol. 2002, 85, 1-13.

[43] Dyck, V.A.; Hendrichs, J.; Robinson, A.S. (Eds.). Sterile insect technique: principles and practice in area-wide integrated pest management. Springer Science \& Business Media. 2006. 
A)

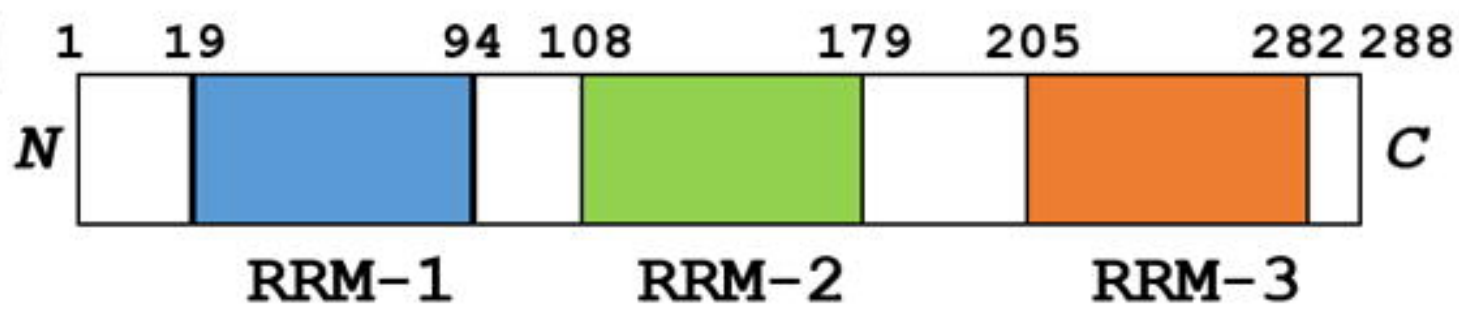

B)

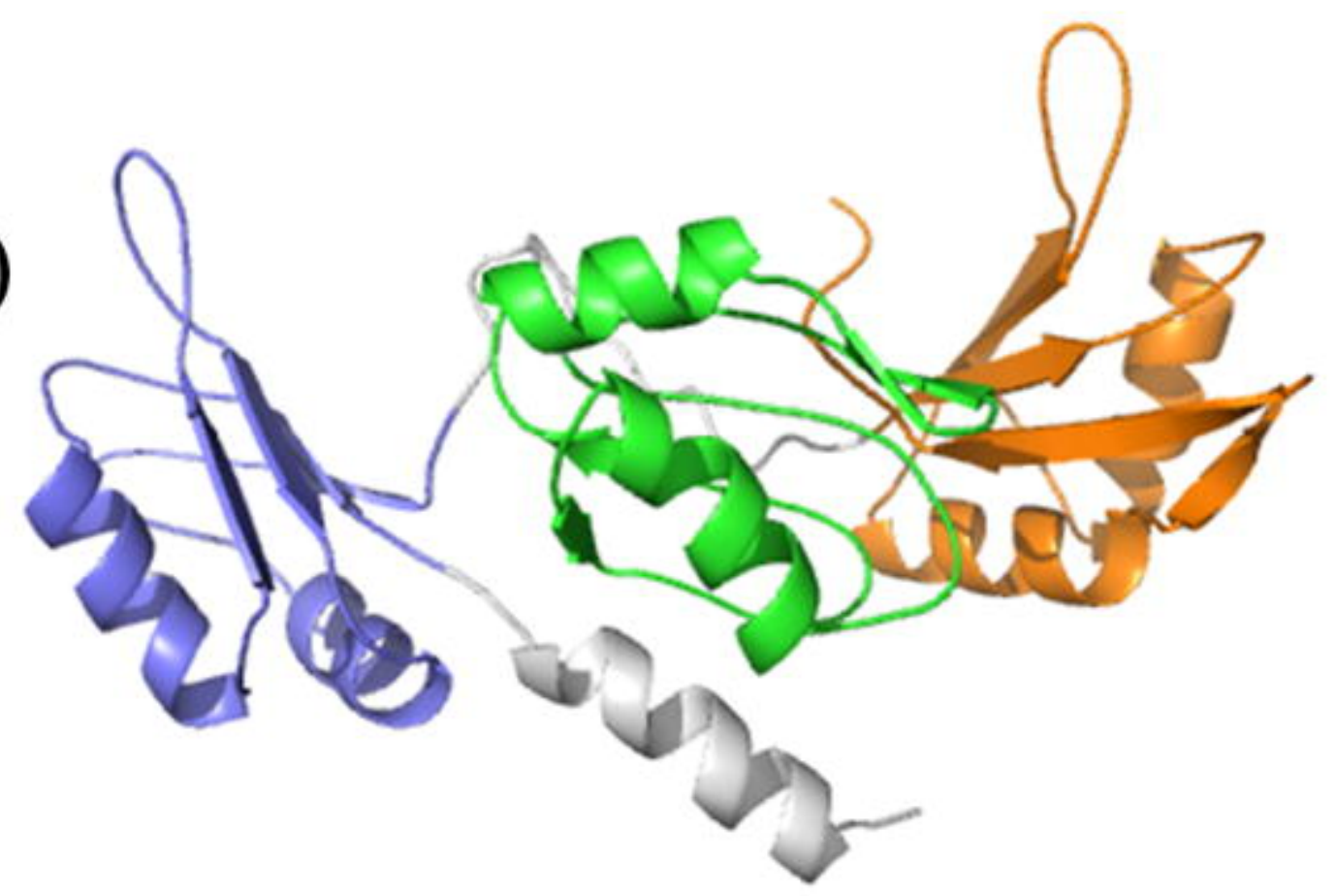

C)

RRM-1 NIX EPABP2 ZCRB1 PABPC1 PrP24

RRM-2

NIX PABPC1 MIP6

NCL PrP24 RNP2 RNP1

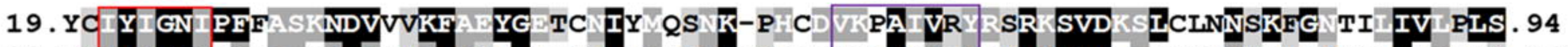
93. RS Y GN D G SIADDIEA TE SCGSINRITILC KFSGHPKGYAYIE AER SVD- AVAMDETVFRGRTIK LPK 168 10. ST Y SN PESL ND YRIE SKG VV TI TMK KDT KSKGVA IL LD DSAON CTRAINNKRLLFGR IKASTAA. 86 11. AS Y GDI HPDV AM YEKR PAGPILSIR CRDMIT RS GYAYVN QPADA MAIDTMNFDVI GKPVRIMWS 87

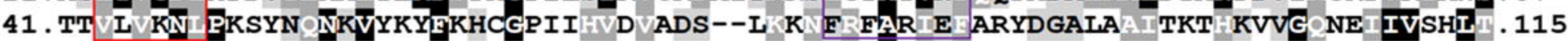

RRM-3

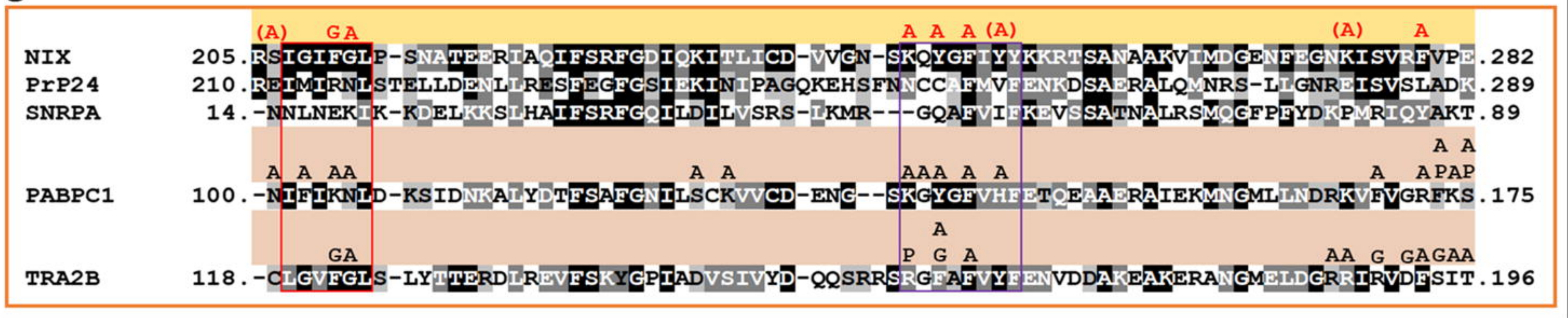

99. IV I KNSCKTSMAELYDEFQKI CPIQN--MFKT----D NMIYINFES-EK M LSLAT P L N NIFKIK V R. 179 313. KIIFIKN-ID SID ALYDTFSAFG IIS-CKVVCD NGS G GEV FE DEAE A BK NG L NDK VG FKS . 175 308. FN FIGNLNP KSVA LKVAISEPFA NDL--AVVDVRTGTN K G VDFE AEDLE A IFLTGLKVIE EIK EKPKG- . 384

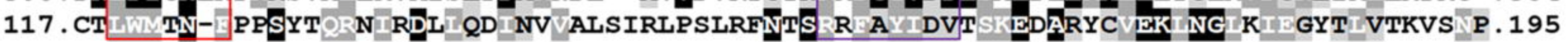
PrP24
210. REIMIRNLSTELLDFNLIRESFEGFGSI
A A AA
A $A$
AAA A A
A $A$
118. -OLGVEGLS

SKOYGETYYYKKRISAA

WNGE -LIGNR ISVSIADK .289 


\section{Figure 2}

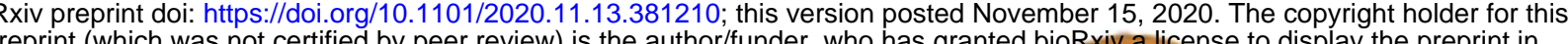

A)

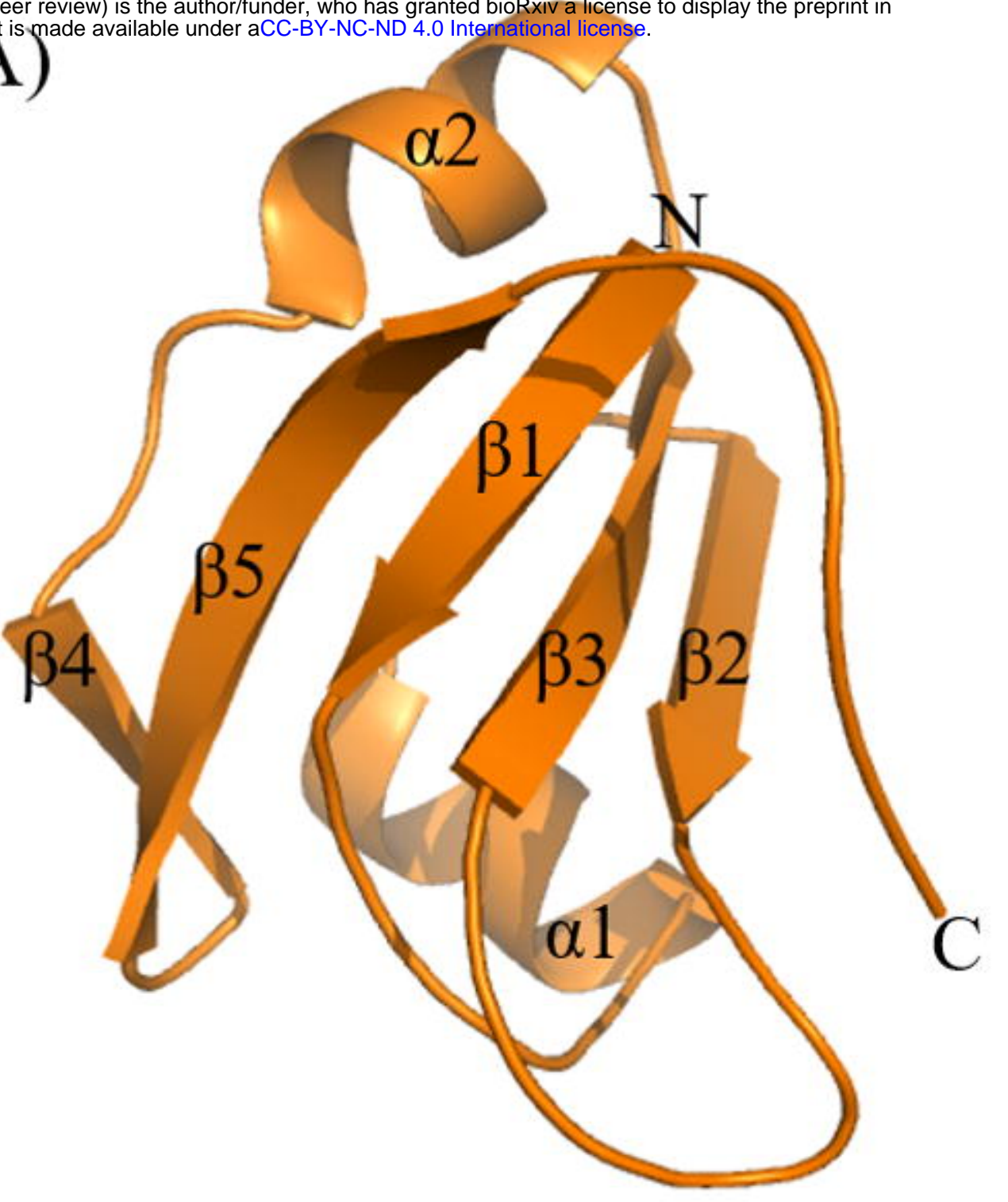

C)

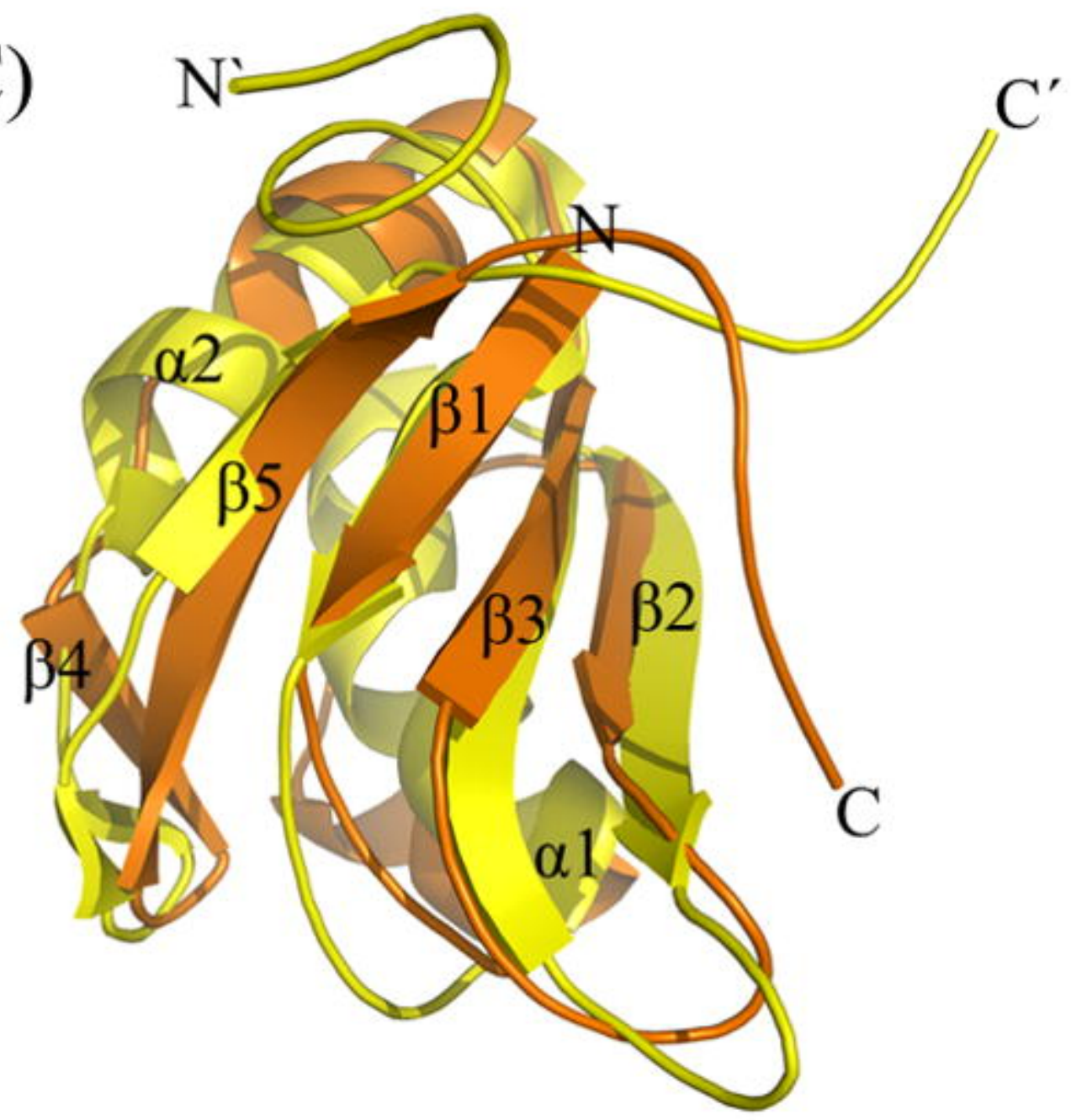

B)

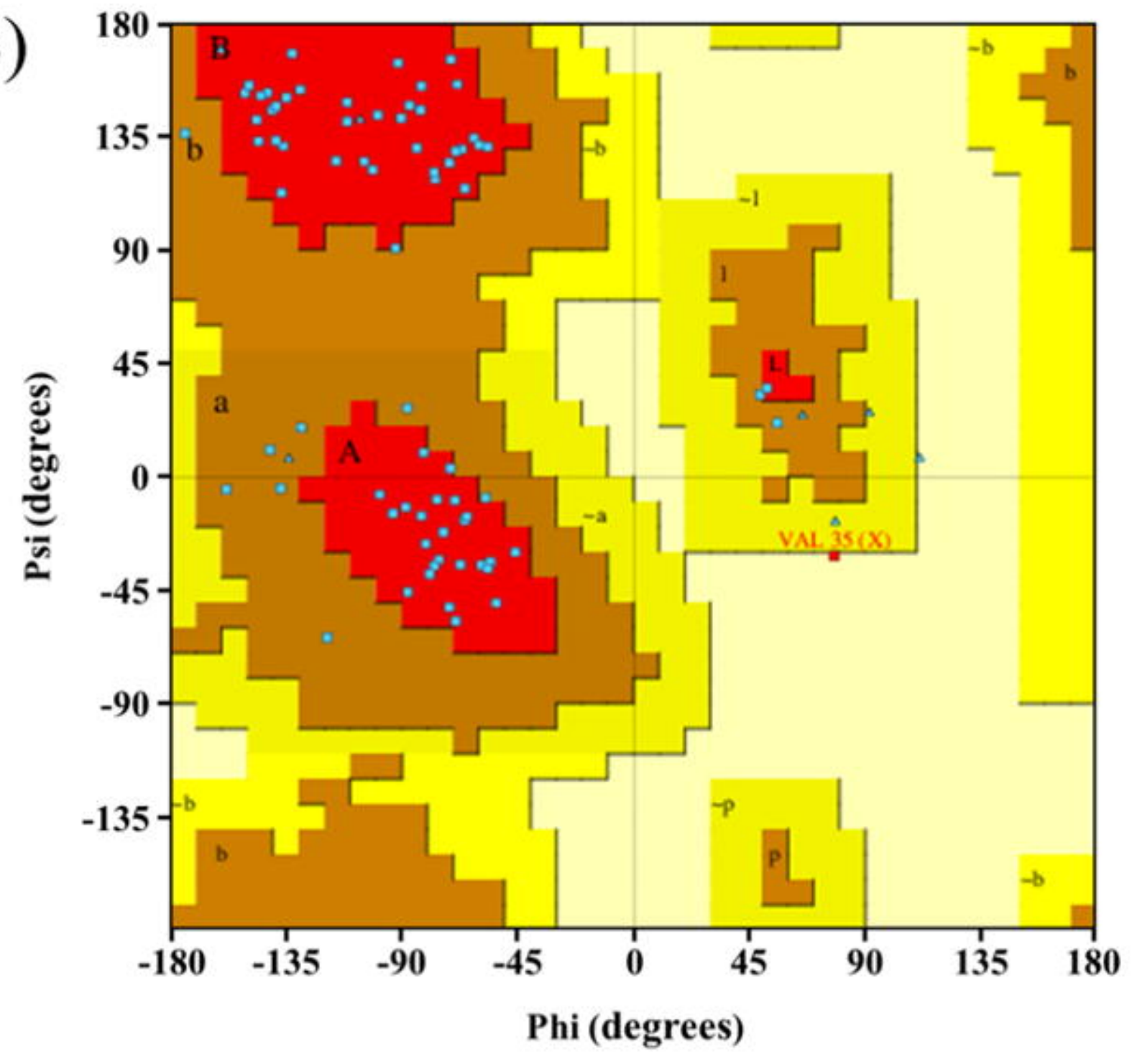

D)

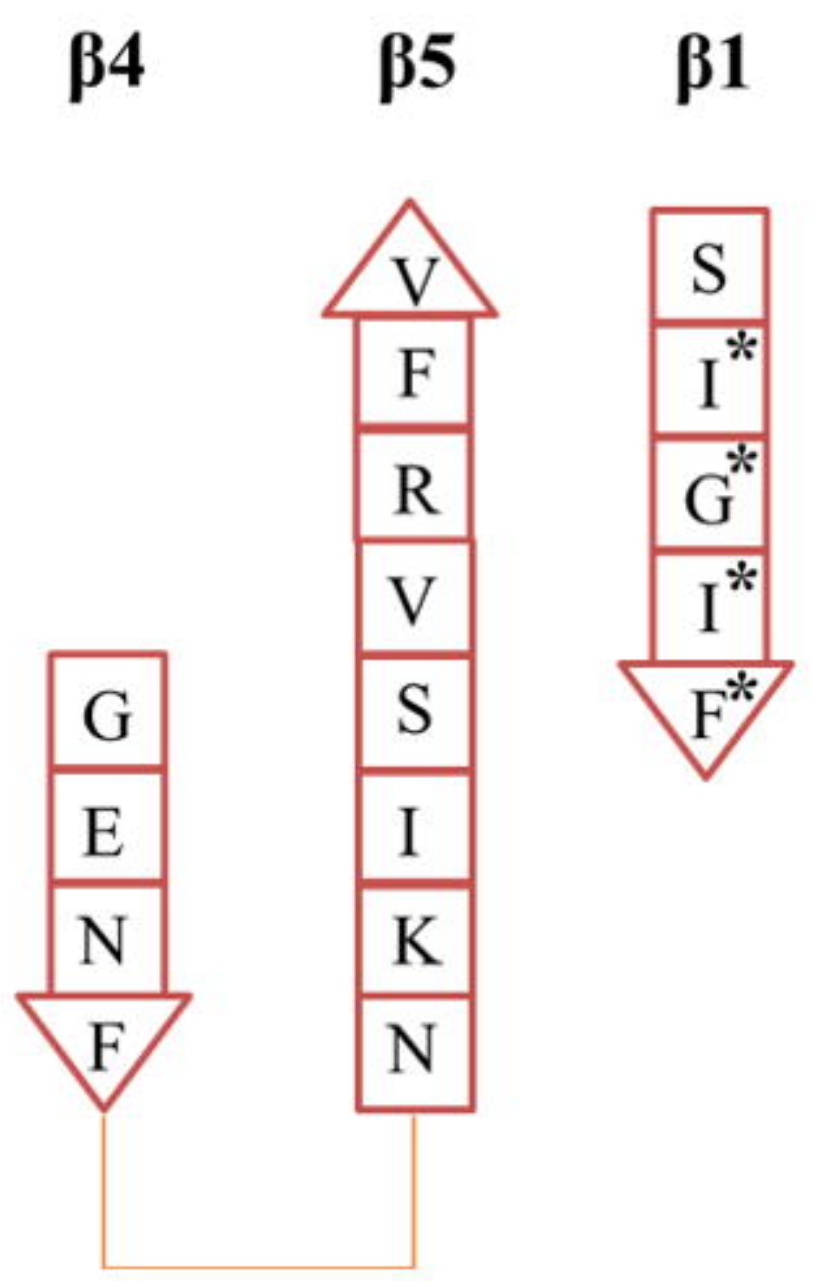

$\beta 3$

$\beta 2$

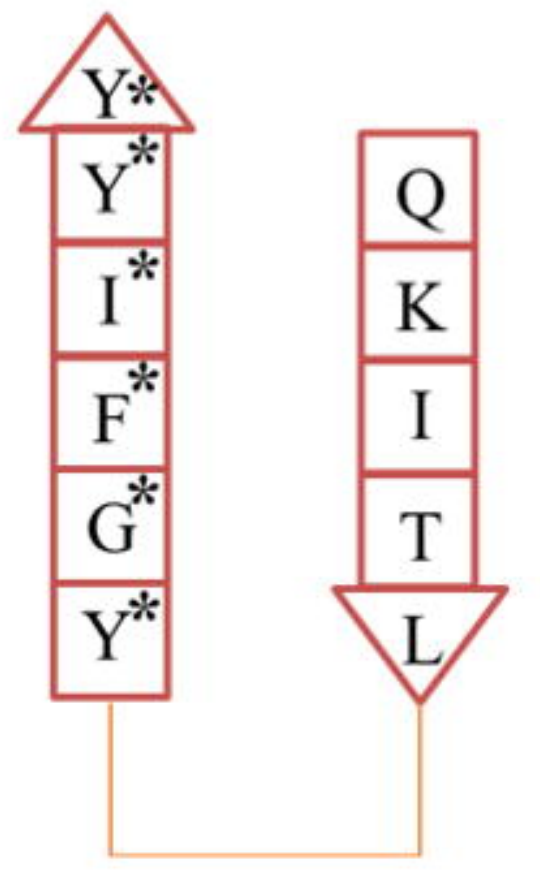




\section{Figure 3}

.

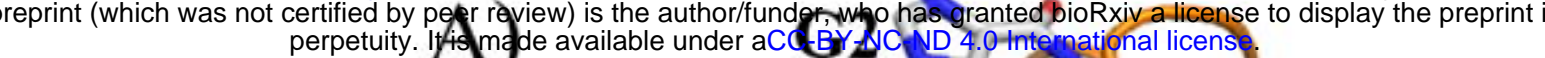

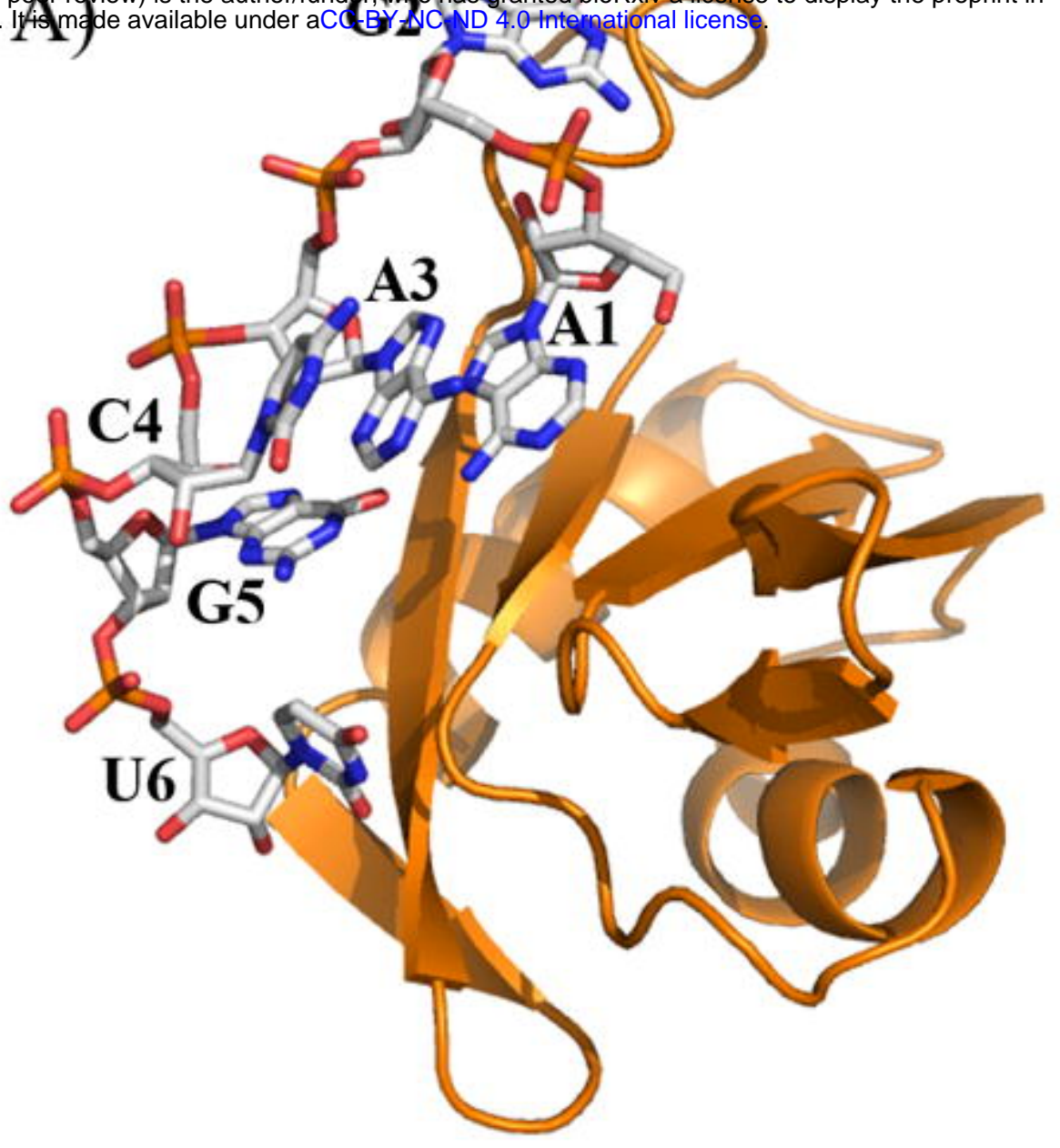

C)

\section{A1}

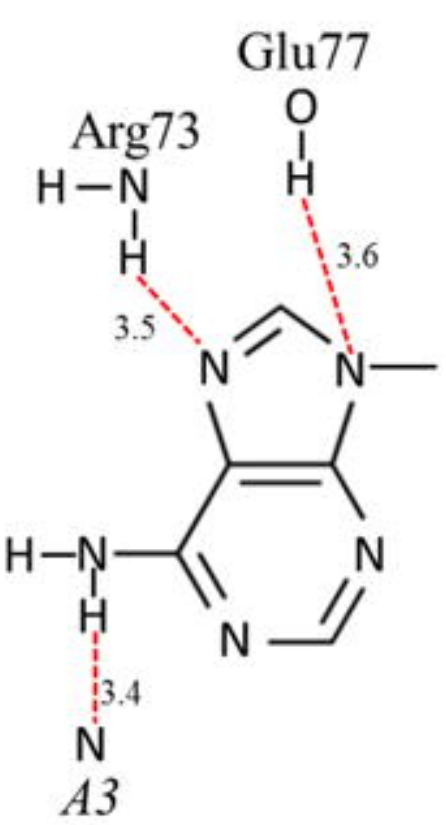

A3

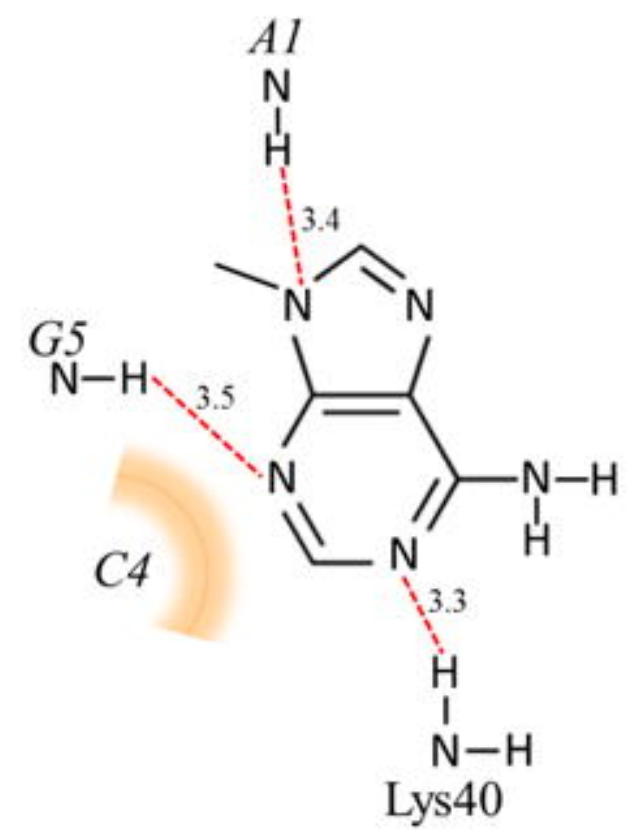

G5

A3 Ribose

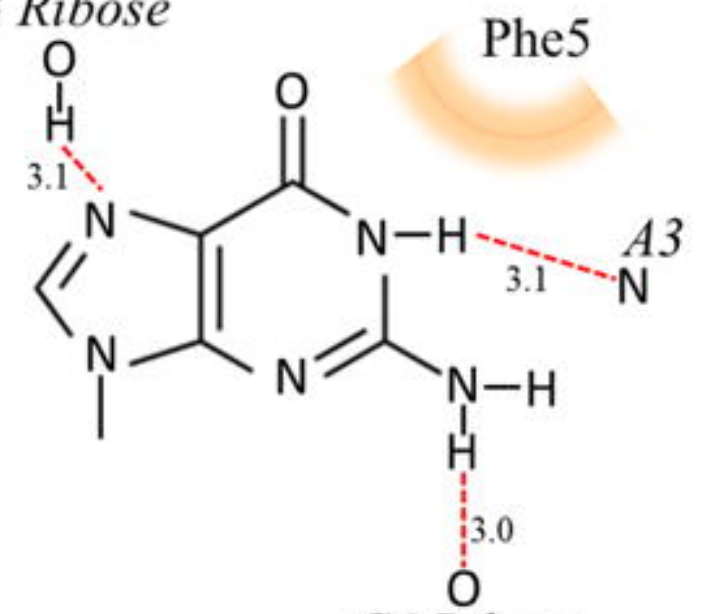

G2

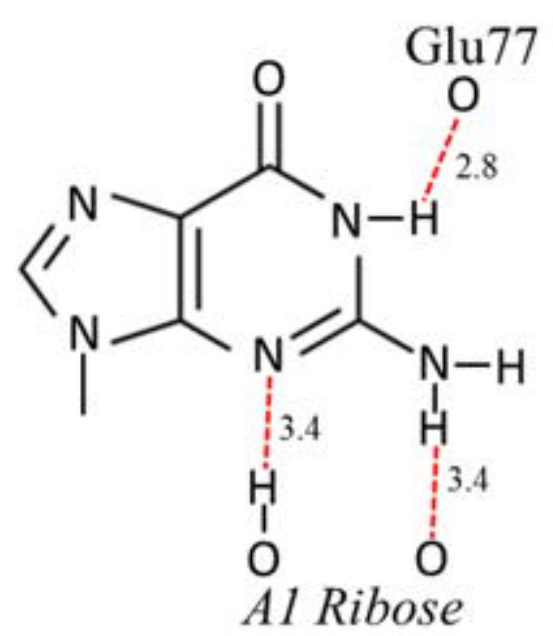

C4<smiles>Cn1ccc(N)nc1=O</smiles>

U6

Phe5

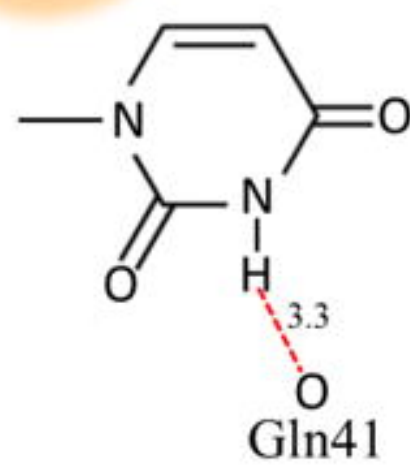


r.

Figure 4

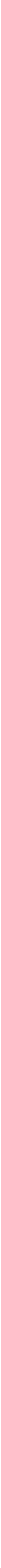


Figure 5

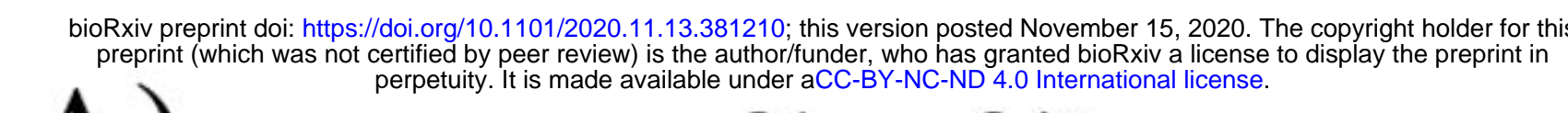

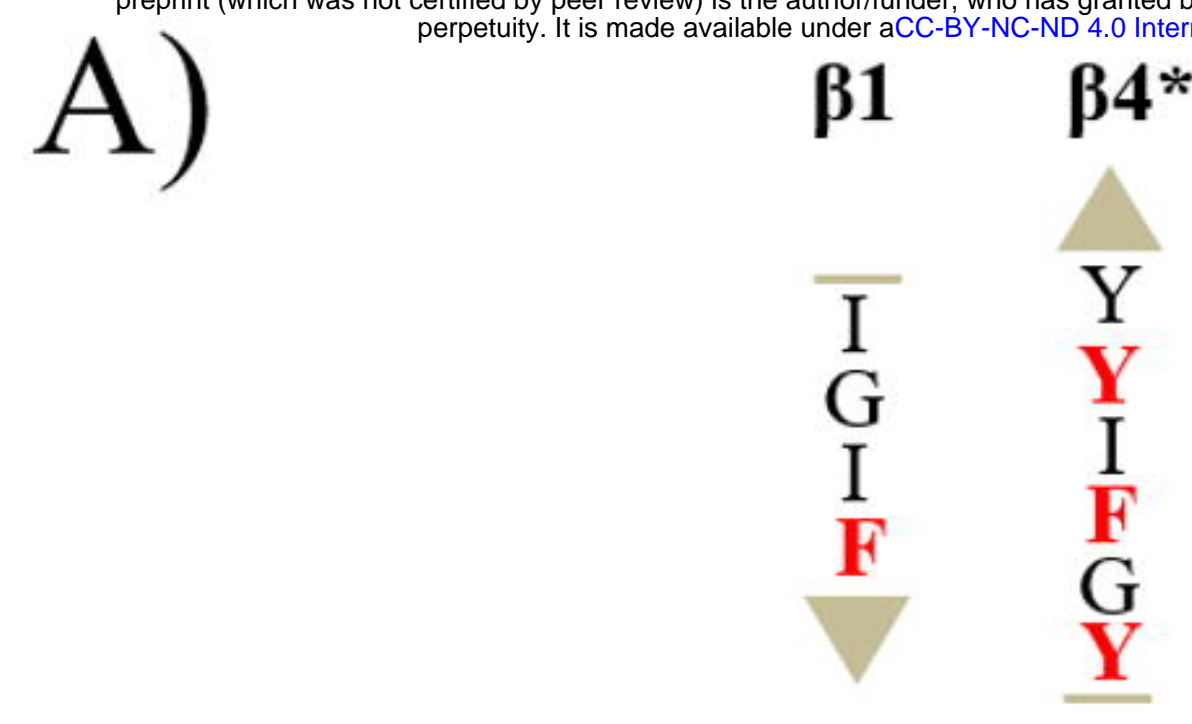

B) $\quad \boldsymbol{\beta 1} \quad \boldsymbol{\beta 3}$
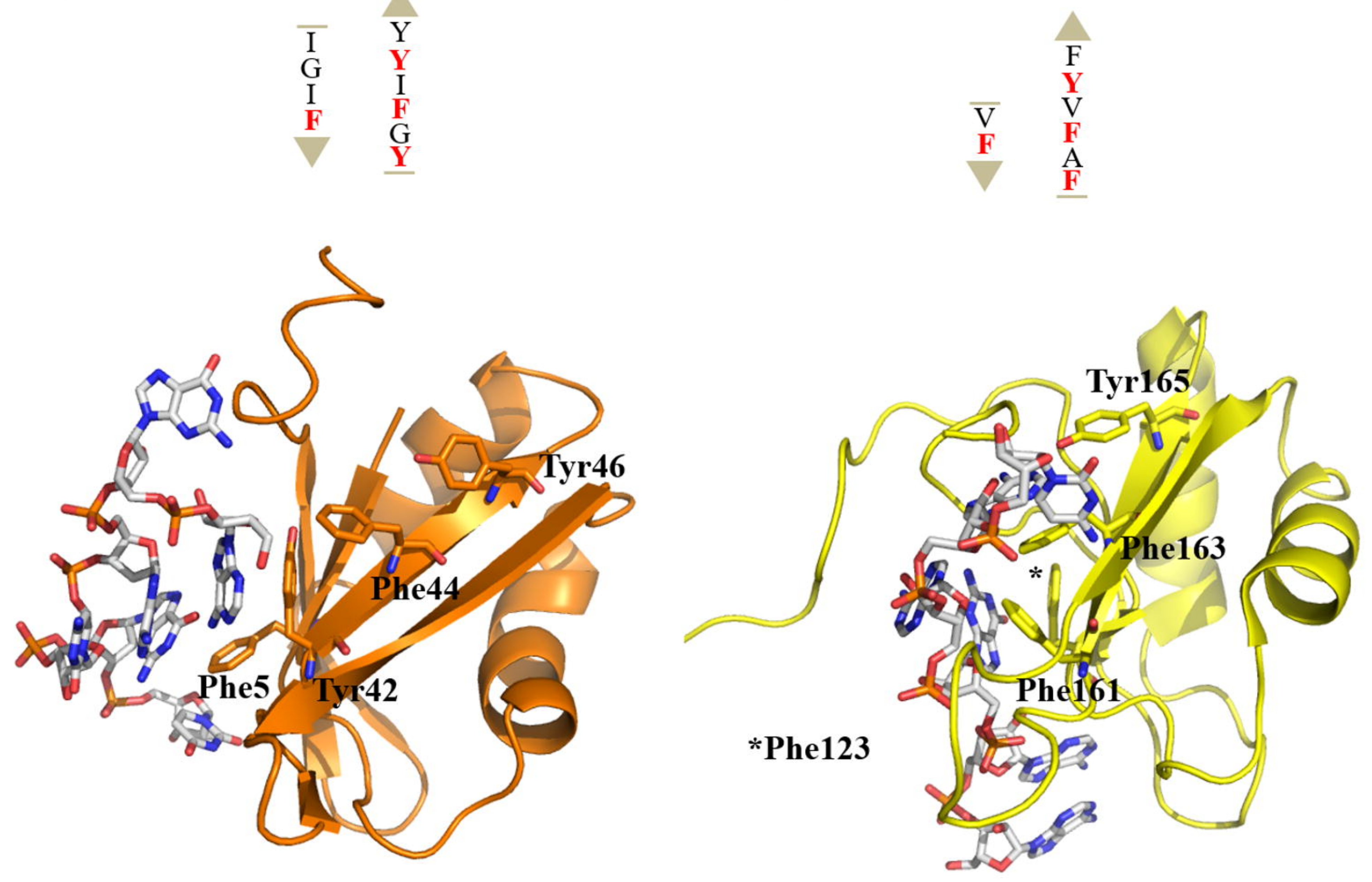
Figure 6

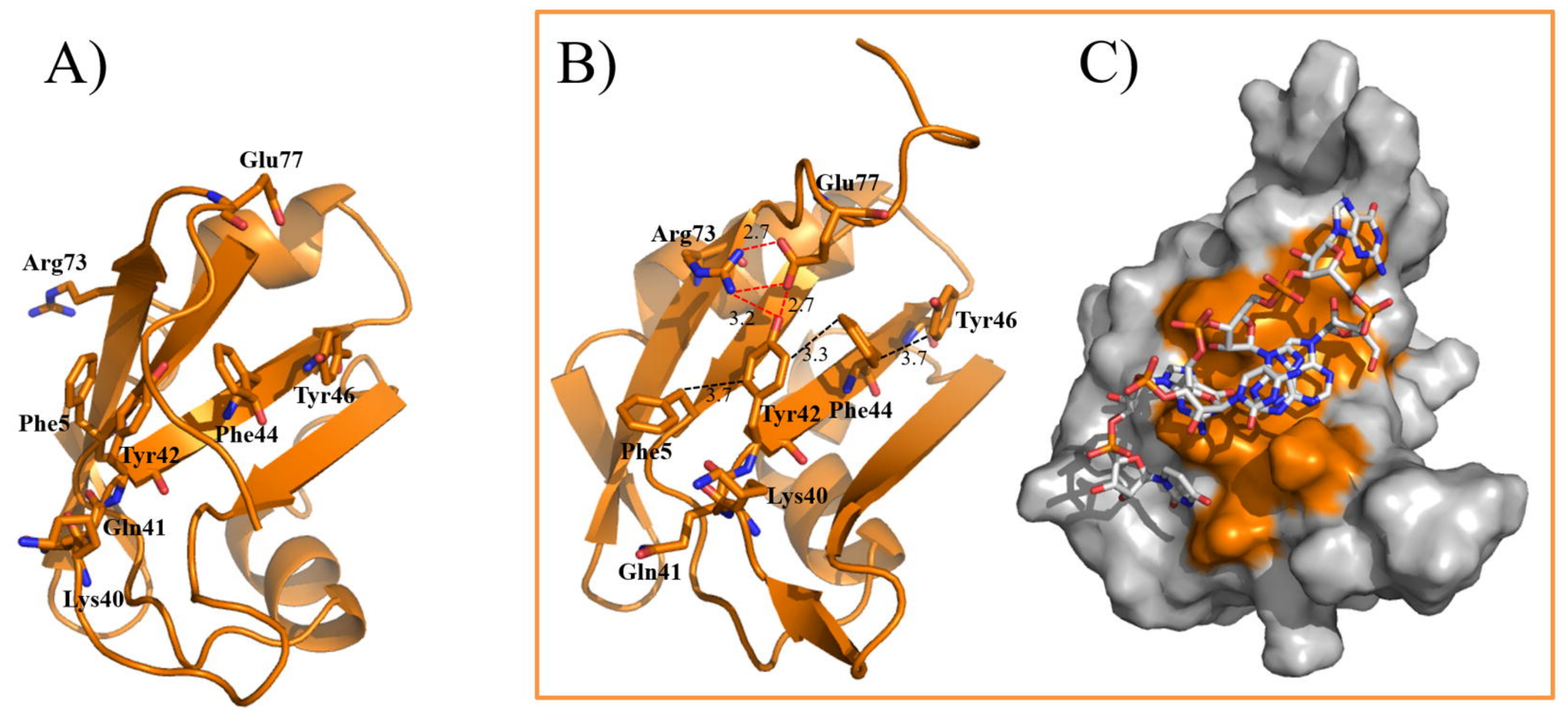



bioRxiv preprint doi: https://doi.org/10.1101/2020.11.13.381210; this version posted November $15,2020$. The copyright holder for this
preprint (which wastopt certified by peer review) is the author/funder, who has granted bioRxiv a license to display the preprint in
perpetuity. It is made avallable under aCC-BY-NC-ND 4.0 International license. 110 perpetuity. It is mad avalable under aCC-BY-NC-ND 4.0 International license.
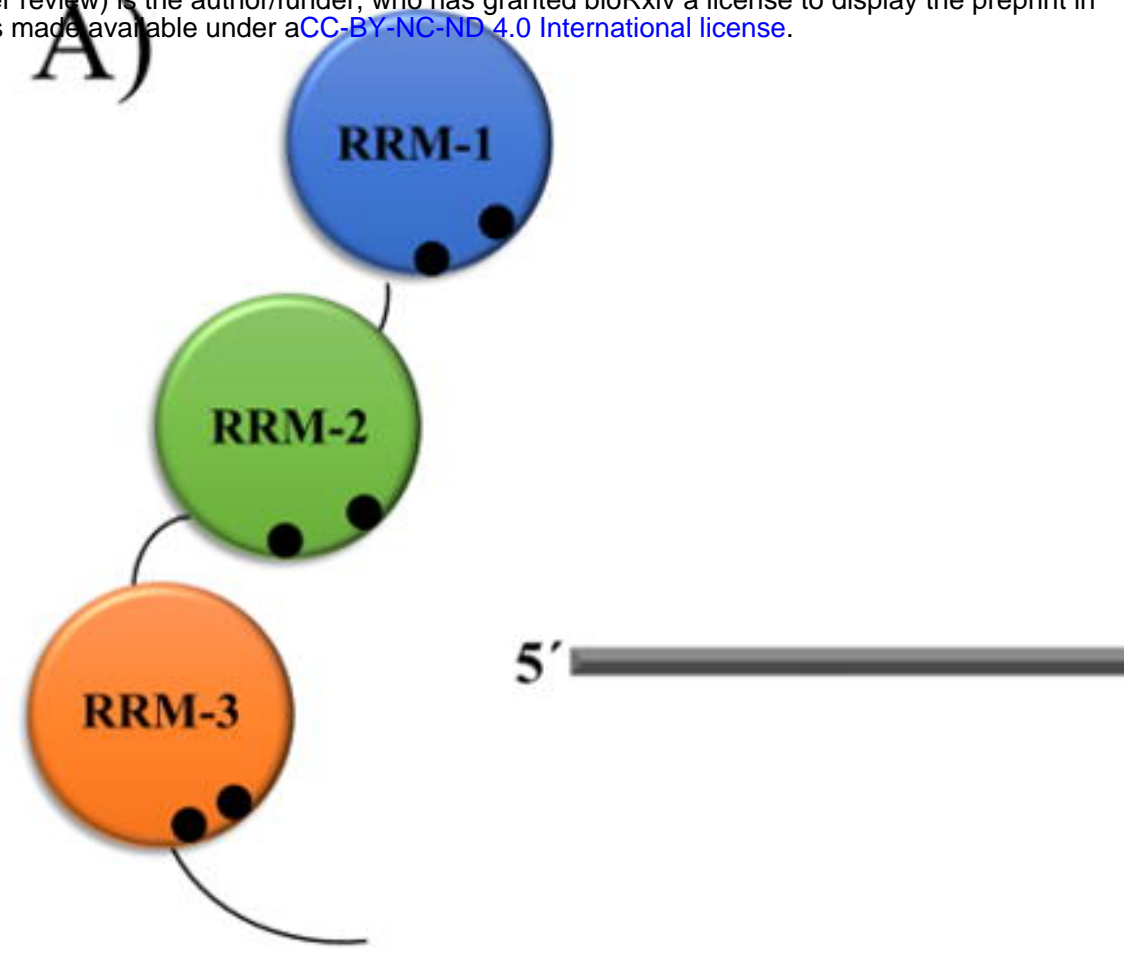

B)

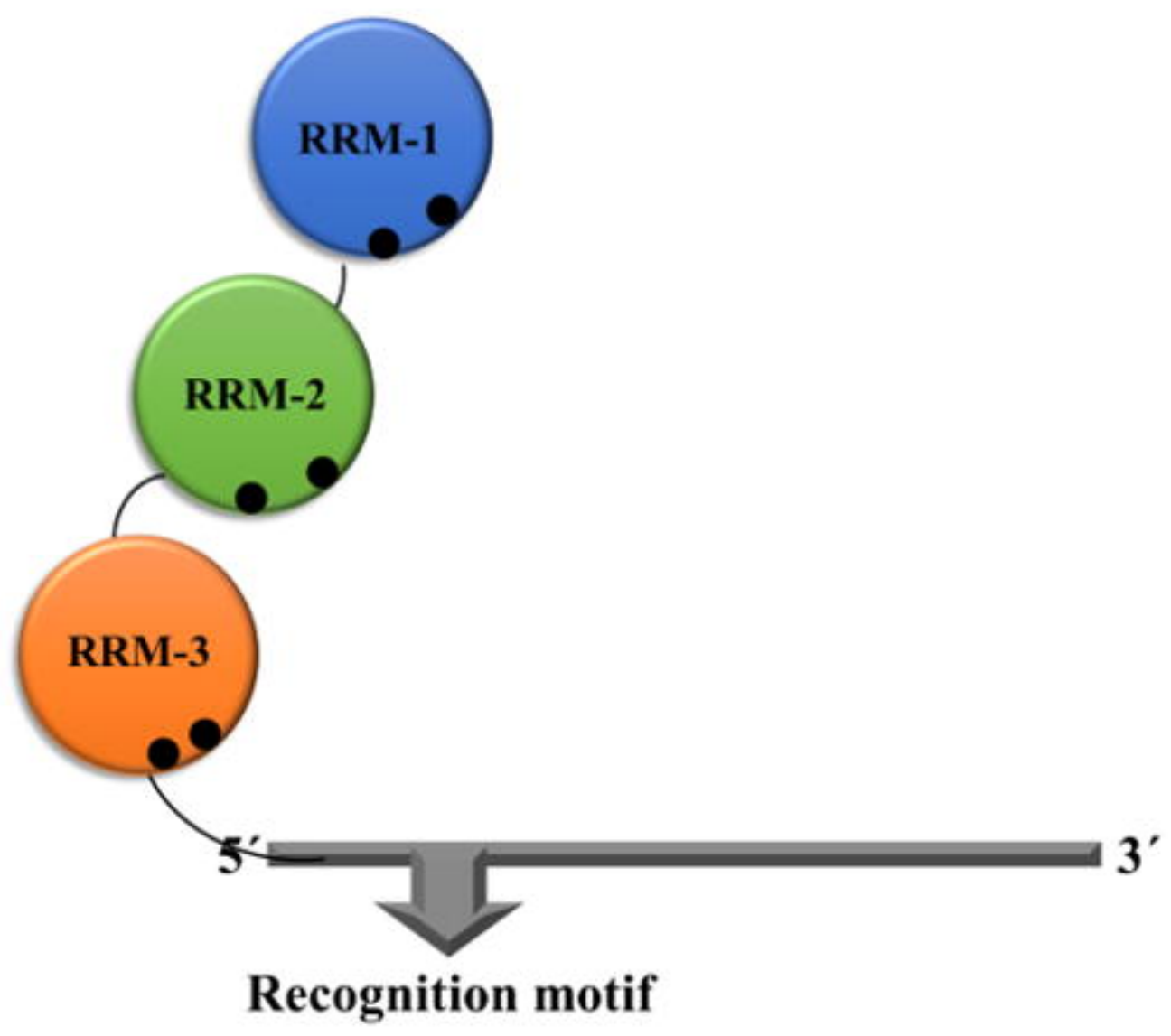

C)

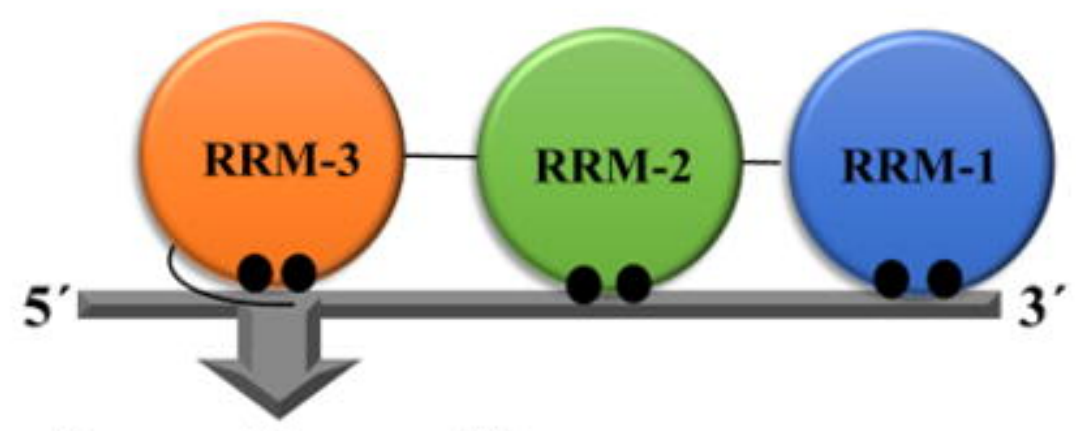

Recognition motif 